Annuaire suisse de politique de développement

\title{
Intégration du secteur privé dans la coopération cantonale genevoise
}

Entretien avec Sylvie Cohen

Sylvie Cohen et Catherine Schümperli Younossian

\section{(Q) OpenEdition}

\section{Journals}

Édition électronique

URL : http://journals.openedition.org/aspd/351

DOI : 10.4000/aspd.351

ISSN : 1663-9669

\section{Éditeur}

Institut de hautes études internationales et du développement

Édition imprimée

Date de publication : 1 octobre 2005

Pagination : 107-114

ISSN : 1660-5934

Référence électronique

Sylvie Cohen et Catherine Schümperli Younossian, «Intégration du secteur privé dans la coopération cantonale genevoise », Annuaire suisse de politique de développement [En ligne], 24-2 I 2005, mis en ligne le 19 février 2010, consulté le 08 septembre 2020. URL : http://journals.openedition.org/aspd/ 351 ; DOI : https://doi.org/10.4000/aspd.351 


\title{
Intégration du secteur privé dans la coopération cantonale genevoise
}

\author{
Entretien avec Sylvie Cohen*
}

En Suisse, les cantons participent également aux efforts d'aide publique au développement. En 2003, les fonds cantonaux attribués à la coopération au développement se sont montés à 18,5 millions de francs, la part genevoise dépassant légèrement 8 millions dévolus aux projets de terrain ${ }^{1}$. Genève est en outre le seul canton à s'être doté, en 2001, d'une loi sur le financement de la solidarité internationale, dans laquelle est inscrit l'objectif d'attribuer 0,7\% du budget cantonal annuel de fonctionnement à la solidarité internationale. Plus récemment, le canton a constitué un groupe de travail chargé de mener une réflexion en vue de créer un nouveau type de partenariat dans le domaine de la coopération au développement avec l'économie privée. Lors d'un entretien accordé à la rédaction de l'Annuaire suisse de politique de développement ${ }^{2}$, Sylvie Cohen, directrice des Affaires extérieures du Département cantonal de l'économie, de l'emploi et des affaires extérieures, a présenté le travail du Service de la solidarité internationale, en charge de la coopération au développement, et a expliqué quelles motivations l'ont conduit à élargir le champ de ses partenaires.

Annuaire Le canton de Genève fait figure de bon élève dlans le domaine de la coopération au développement. Comment, historiquement, son engagement s'est-ill construit, et sur quel type de partenariat?

Sylvie Cohen La politique de coopération au développement, introduite progressivement au début des années 1960 par le canton de Genève, s'est toujours appuyée sur une forme de partenariat public-privé, mais avec le soutien des associations genevoises de développement (PPPa, «a» désignant les associations). En effet, sans intervenir directement dans les pays en développement, il a consacré une ligne budgétaire pour l'appui à des projets présentés par différentes associations, fondations ou ONG, actives dans ce domaine. Entre 1962 et 1966, un crédit de 1,25 million de francs a été voté par le Grand Conseil en faveur de la coopération technique au développement. En 1967, ce crédit s'est mué en une subvention annuelle de 500'000 francs qui a progressivement atteint le montant de 2,3 millions en 2000. A cette date, l'abolition du droit des pauvres, qui servait en partie à financer l'aide humanitaire, a incité le Conseil d'Etat à ajouter 1 million de francs à cette somme, l'aide humanitaire et la coopération au développement étant ainsi regroupées sous la même rubrique budgétaire. Déjà à cette époque, la Fédération genevoise

* Directrice des Affaires extérieure du canton de Genève.

1 Source: DDC, Aide de la Suisse aux pays en développement et aux pays en transition. Statistiques 2003, Berne, DDD, 2005, p. 135.

2 Propos recueillis par Catherine Schümperli Younossian en avril 2005. 
de coopération (FGC), qui rassemble une cinquantaine d'associations, figure comme un partenaire important pour le canton puisqu'une convention, signée en 1998, lui accorde les deux tiers des 2,3 millions de francs destinés à la coopération au développement. Ainsi s'est instauré un partenariat entre le secteur public et des acteurs privés du monde associatif.

En 2001, le canton de Genève s'est doté d'une nouvelle loi relative au financement de la coopération au développement. Quelles sont les spécificités de ce texte?

Le 5 octobre 2001, le Grand Conseil genevois a voté une nouvelle loi «sur le financement de la solidarité internationale», selon laquelle le canton devrait consacrer $0,7 \%$ de son budget annuel de fonctionnement à des actions de solidarité avec les pays en développement. Mais, au-delà de la volonté d'augmenter quantitativement les moyens à disposition pour l'aide au développement, il s'agissait aussi de réfléchir à de nouvelles formes de coopération. Ainsi, la Direction des affaires extérieures s'est vue chargée d'élaborer un document de référence visant à introduire le concept de développement durable dans la politique cantonale de coopération au développement. La réflexion menée par un groupe de travail, constitué à cet effet, a donc largement servi à nourrir les travaux qui ont abouti à la publication d'un règlement d'application de la nouvelle loi sur le financement de la solidarité internationale, entrée en vigueur le $1^{\text {er juillet } 2002 .}$

$\mathrm{Si}$ le règlement d'application de la nouvelle loi intègre les principes du développement durable, il ne marque pas d'évolution notable dans le domaine du partenariat public-privé. Le financement de la solidarité internationale s'opère selon des critères «classiques»: approche par projet, domaines d'action définis hors du champ économique (excepté quelques activités artisanales génératrices de revenus), collaboration reposant sur le savoir-faire d'un partenaire genevois issu des milieux associatifs ou des ONG.

Quelles sont alors les nouvelles formes de coopération introduites par le règlement d'application de la loi de 2001?

Deux nouveautés ont été introduites par le règlement d'application, mais elles n'intègrent pas encore le secteur privé dans la coopération cantonale au développement; ce pas supplémentaire se fera dans un deuxième temps. La première concerne un élargissement de la définition de la solidarité internationale, qui dépasse le strict cadre de la coopération au développement et englobe les actions menées à Genève en faveur des pays en développement. Cet aspect concerne essentiellement des actions liées à la Genève internationale: promotion des droits de l'homme, participation à des manifestations telles que le Forum mondial de la société civile, etc. La seconde consacre la notion de «coopération décentralisée », qui permet à l'Etat de Genève de mener directement, sur le terrain, des actions de coopération au développement, en collaboration avec des collectivités locales.

$\mathrm{Ni}$ le législateur, ni les partenaires de la solidarité internationale ne semblaient pousser à explorer de nouvelles voies dans la coopération au développement. C'est en définitive l'Agenda 
21 cantonal qui sera l'élément déclencheur d'une réflexion plus novatrice.

Quelles ont été les motivations du Département de l'économie, de l'emploi et des affaires extérieures pour envisager des collaborations avec le secteur privé dans le domaine de la coopération au développement?

Dans son nouveau calendrier de législature 2003-2006, la Loi sur l'action publique en vue d'un développement durable prévoit à nouveau un volet «coopération au développement». Après l'adoption du Pacte mondial par les Nations unies, la Conférence sur le financement du développement de Monterrey (Mexique) et le Sommet mondial sur le développement durable de Johannesburg montraient clairement la nouvelle tendance prise pour atteindre les objectifs de développement de l'ONU. Sentant le besoin de faire évoluer dans ce sens la politique cantonale de solidarité internationale, mon département a donc proposé d'explorer de nouvelles pistes dans le domaine de la coopération économique et commerciale, en introduisant la possibilité de d'établir des partenariats entre les pouvoirs publics et l'économie privée.

L'idée ayant été acceptée par le comité interdépartemental de l'Agenda 21, le Service de la solidarité internationale a mis sur pied un groupe de travail regroupant des acteurs de l'administration cantonale, de la Direction du développement et de la coopération (DDC), de l'ONU, du monde associatif et de l'économie privée. Ce groupe a eu à répondre à une question préliminaire: L'Etat de Genève doit-il élargir le champ d'intervention de la solidarité internationale à une coopération écono- mique et commerciale, en introduisant des partenariats public-entreprises?

En clair, il s'agissait non seulement d'apprécier l'opportunité d'introduire ou non de nouvelles approches dans la politique cantonale de solidarité internationale, mais aussi d'évaluer le rôle qu'un canton peut jouer dans le financement d'une coopération au développement ouverte à des partenariats avec l'économie privée.

\section{Quels ont été les points essentiels de convergence et de divergence lors des discussions au sein du groupe de travail?}

Un point d'accord s'est rapidement esquissé: avec des compétences restreintes en matière de collaboration internationale, avec des moyens financiers relativement limités (environ 8 millions de francs dévolus au cofinancement d'actions de terrain en 2004), le canton de Genève n'avait pas d'autre choix que de limiter, de façon réaliste, le champ de son intervention dans le domaine de la coopération économique, en balisant par des gardefous les partenariats possibles entre le secteur public et l'économie privée. Pas question pour lui de «jouer dans la même cour» que la Confédération, qui participe pleinement aux stratégies multilatérales de développement. Il a donc été décidé de laisser à la DDC et au Secrétariat d'Etat à l'économie (seco) l'aptitude d'intervenir au niveau macroéconomique et de situer celle du canton au niveau microéconomique. Ainsi, l'idée de créer un fonds cantonal pour la coopération économique destiné à financer des partenariats public-entreprises a été d'emblée écartée par le groupe de travail, pour des raisons de financement (la coopération au développement économique devant 
s'inscrire dans le budget global de la solidarité internationale) et parce qu'un tel fonds nécessiterait la création d'une structure ad hoc ou d'une «externalisation», rendant les contrôles plus difficiles.

Après d'intenses discussions, le groupe de travail a finalement défini deux espaces d'intervention possibles pour le canton: d'une part, le renforcement du tissu économique local des pays en développement (y compris par un soutien au commerce équitable Sud-Nord et Sud-Sud), et, d'autre part, le transfert de savoirs, de savoir-faire et de technologies, l'Etat de Genève jouant un rôle de facilitateur pour des mises en contact entre partenaires (entreprises genevoises, ONG, responsables de projets dans les pays en développement, etc.) ainsi que de bailleur de fonds pour aider financièrement à la réalisation du projet.

Quelles sont les règles du jeu définies pour que les partenariats publicprivé, avec les entreprises, puissent être mis en ouvre avec succès?

Afin de ne pas dénaturer l'esprit de la Loi sur le financement de la solidarité internationale, il devait être clair que tout projet de coopération économique ou commerciale, présenté par des entreprises genevoises, bénéficierait nécessairement aux populations des pays en développement (ou émergents), en fonction d'un besoin local exprimé par les partenaires du terrain. Si l'entreprise peut en tirer un profit, son action doit se distinguer d'une opération commerciale habituelle, en démontrant les bénéfices sociaux, économiques et environnementaux qu'elle apporte à la collectivité, sur le terrain. Ce principe permet d'éviter les distorsions de concurrence induites par une intervention du secteur public. En outre, l'opération ne doit pas aboutir à un transfert de postes de travail du Nord au Sud. Aucun projet visant à des délocalisations ne saurait être éligible.

Le groupe de travail chargé de réfléchir au volet «coopération au développement économique et commercial/ PPP» de l'Agenda 21 cantonal a produit un document de référence ${ }^{3}$, qui a été validé par le comité interdépartemental de l'Agenda 21 et, en novembre 2004, par le Conseil de l'environnement, où siègent des représentants de l'Etat et des acteurs de la société civile. Les principes émis dans ce texte seront intégrés dans le Règlement d'application de la Loi sur le financement de la solidarité internationale, qui, d'ici à la fin 2005, doit être renouvelé et modifié à la lumière d'une expérience pratique de trois ans.

Le document, Agenda 21. Document de référence de l'Etat de Genève. Coopération au développement économique, définit clairement le champ d'action pour les PPPe («e» désignant les entreprises), mais surtout les critères d'éligibilité des partenaires et des projets. Etant donné les moyens financiers susceptibles d'être mobilisés et le champ d'intervention défini, les porteurs de projet issus de l'économie privée devraient être généralement des PME. L'entité requérante - outre les garanties éthiques qui lui seront demandées devra justifier d'activités à Genève. Par ailleurs, sa fortune sera examinée pour déterminer la pertinence de la

3 Agenda 21. Document de référence de l'Etat de Genève. Coopération au développement économique. Ce document est publiée en annexe. 
demande. Il apparaît ainsi clairement que le profil d'une multinationale serait difficilement compatible avec ces exigences.

Pensez-vous que le secteur privé se montrera intéressé par ce nouvel instrument de la coopération cantonale en faveur du développement?

Le processus que nous avons engagé avait pour but de réfléchir aux nouveaux instruments de la coopération internationale au développement, tels que le partenariat avec le secteur privé, et de rendre possible leur mise en œuvre au niveau d'un service cantonal de solidarité internationale, selon des critères précis. Nous ne cherchons pas à convaincre des entreprises de faire de la coopération, mais, lorsque certaines d'entre elles mani- festent le désir de s'engager dans cette voie, nous souhaitons pouvoir encourager de telles actions. Le Service de la solidarité internationale a approuvé un premier projet de PPPe, qui remplit tous les critères établis dans le document de référence Agenda 21. Coopération au développement économique. Il s'agit de la construction d'un centre de formation visant à promouvoir des techniques de construction écologiques et traditionnelles au Burkina Faso (encadré). Gageons que d'autres acteurs du secteur privé manifesteront leur intérêt à se lancer dans de fructueux projets de coopération au développement.

\section{Site Internet}

Service de la solidarité internationale de l'Etat de Genève : <www.geneve.ch/solidarite>. 


\section{Centre de promotion de la construction en terre, Burkina Faso Exemple d'un partenariat public-privé soutenu par le canton de Genève}

Le projet de la création d'un "Centre de formation, d'expérimentation et de promotion des technologies de la construction en terre" est le premier exemple de partenariat public-privé auquel participe le canton de Genève. Ce projet est intéressant à plus d'un titre, d'une part à travers les objectifs qu'il poursuit en termes de développement durable et de formation, mais d'autre part également par la nature des relations entre les partenaires, locaux et suisses, afin de le mener à bien.

Le projet démarre à l'initiative d'une association burkinabé, I'Association pour l'habitat en terre (AHT), qui souhaite promouvoir la construction d'habitations selon des techniques traditionnelles, en prenant en compte la préservation de l'environnement (notamment en diminuant l'utilisation du bois dans la construction) et en mettant en valeur l'architecture et les techniques de construction locales. Pour réaliser ses objectifs, I'AHT a souhaité se doter d'un centre de formation et d'expérimentation dédié aux constructions en terre.

La coopération suisse, en collaboration avec le Burkina Faso, a lancé dans les années 1980 le Programme de promotion de l'artisanat au Burkina (PAB), qui contribue à la promotion des micro et petites entreprises du secteur de l'artisanat utilitaire. En 1995, en coordination avec d'autres agences de coopération et sous l'impulsion du PAB, une cellule d'appui à la formation professionnelle est créée. La dimension opérationnelle du PAB est confiée à un bureau d'étude suisse, AE Echanges SA.

L'origine du soutien genevois au projet revient à la Société suisse des entrepreneurs, section Genève (SG/SSE), association professionnelle qui, dans le cadre de son centième anniversaire, a souhaité participer à un projet de développement.

Le projet comporte trois volets essentiels:

- la réalisation des infrastructures du centre de promotion de la construction de la terre, sur la commune de Réo;

- la promotion de la construction en terre (formation des apprentis locaux, perfectionnement, appuiconseil aux constructeurs et promoteurs de cette technique);

ـ l'envoi d'apprentis genevois au Burkina Faso dans le cadre de leur formation.

Pour mener à bien ce projet, divers partenaires sont impliqués dans sa réalisation. L'AHT est le maître d'ouvrage et le responsable de la construction du centre, ainsi que de l'organisation des programmes de formation. En été 2005, la construction du centre approche de son achèvement et son inauguration est prévue pour la fin de l'année. La SG/SSE est le partenaire financier de I'AHT et participe à la conception générale du projet. Elle a contribué au financement de la construction du centre à hauteur de 200'000 francs suisses, dont 80'000 francs ont été financés par l'Etat de Genève. Elle collabore en outre à la vie du centre en organisant des stages - et en participant à leur financement - pour des apprentis maçons genevois. Depuis 2003, elle a ainsi envoyé chaque année trois groupes de vingt apprentis, le dernier stage à ce jour datant de février 2005. La commune de Réo a mis à disposition le terrain pour la construction du centre et apporte un soutien administratif et politique au projet. Projet qui s'inscrit dans le cadre du PAB et dont le suivi est confié au bureau d'étude mentionné plus haut. 


\section{Annexe}

\section{AGENDA 21 \\ Document de référence de l'Etat de Genève. Coopération au développement économique}

\section{Préambule}

Conformément au Règlement d'application de la Loi sur le financement de la solidarité internationale (D 106.01 du 1er juillet 2002 - art.2), I'Etat vise, par son action, à contribuer à la promotion de la paix, à la lutte contre la pauvreté dans les pays les moins favorisés et à réduire les phénomènes d'inégalités socio-économiques, les discriminations, les exclusions et les violations des droits humains, la dégradation de l'environnement et le gaspillage des ressources naturelles dans la perspective d'un développement durable.

Le soutien accordé dès lors à des associations ou institutions a permis la mise en œuvre de nombreux projets, notamment de coopération technique au développement, de coopération dans les domaines du social et de la santé, dans le domaine de l'environnement et de la défense des droits humains, à l'instar du renforcement de l'accès à l'information, de la culture, de la citoyenneté et de la bonne gouvernance.

La coopération dans le domaine économique s'est concentrée sur des projets concernant des activités artisanales et agricoles, sur l'appui au développement d'activités visant l'autosuffisance alimentaire, ou encore au renforcement de l'accès aux technologies de l'information.

Le partenariat public-entreprises n'est cependant pas pris en compte par le règlement et les textes de références actuellement en vigueur, qui stipulent que l'aide apportée par l'Etat ne peut être adressée qu'à des associations à but non lucratif.

L'évolution des politiques de lutte contre la pauvreté suscitées par l'Organisation des Nations unies incite aujourd'hui à élargir, à certaines conditions, l'approche cantonale de la coopération au développement économique et commercial, susceptible d'être mise en place sur la base d'un partenariat public-entreprises. Dans ce domaine l'engagement de l'Etat de Genève se veut subsidiaire aux actions de la DDC et du seco.

Les projets soutenus devront faire preuve de leur respect des principes du développement durable par l'attention portée aux bénéfices sociaux, économiques, et environnementaux pour la communauté dans laquelle ils s'insèrent.

\section{Champ d'action}

L'Etat devrait étendre son appui aux besoins exprimés, en soutenant les actions et projets présentés par des entreprises genevoises et qui dépassent leur cadre opérationnel habituel. Le soutien financier de l'Etat de Genève pourrait intervenir dès lors que des bénéfices sociaux, économiques et environnementaux sont démontrables.

Ces projets devront se référer aux champs d'action suivants:

- renforcement du tissu économique local;

- transfert de savoirs, de savoir-faire et de technologies.

Ils devraient viser in fine à améliorer les conditions de vie des communautés locales concernées.

Dans cette perspective, I'Etat facilite la mise en contact d'entités potentiellement intéressées.

\section{Porteur de projet}

En règle générale, l'Etat peut répondre aux actions et projets présentés par des entreprises genevoises inscrites au Registre du commerce et/ou qui justifient d'une activité régulière à Genève et qui ont des projets dont les objectifs sont de favoriser le développement et le renforcement du tissu économique des pays défavorisés.

Les partenariats entre les entreprises et le secteur associatif sont encouragés.

Le porteur de projet doit pouvoir démontrer sa stabilité financière et s'engage à assumer la responsabilité opérationnelle, administrative et technique du projet dont il a la charge. II met également à disposition les ressources et compétences nécessaires. 


\section{Critères}

ـ Eligibilité des partenaires

- Partenaires porteur de projet

Les partenaires porteur de projet doivent satisfaire aux conditions suivantes:

- être inscrits au Registre du commerce et/ou actifs à Genève;

- être à jour dans le paiement des cotisations sociales;

- faire preuve de leur stabilité financière;

- justifier de compétences et d'expériences dans le domaine d'intervention du projet;

- s'engager à assurer une totale transparence dans la provenance et l'usage des fonds utilisés dans le cadre du projet;

- adhérer à une charte de bonne conduite;

- disposer d'un partenariat solide avec des acteurs locaux.

- Partenaires locaux

Les partenaires locaux peuvent être des

- collectivités locales;

- institutions faîtières ou associations professionnelles;

- entreprises locales établies ou en création, regroupement d'entreprises;

- ONG.

Le dossier doit comprendre une présentation de la ou des entités locales concernées: identités des responsables, buts poursuivis, compétences et spécialités, engagement et/ou pièces certificatives.

口 Eligibilité des projets

- Orientation

L'orientation du projet doit dépasser le cadre opérationnel habituel du porteur de projet, dans la mesure où un financement public d'une opération privée de solidarité internationale se distingue d'une opération purement commerciale.

- Contribution attendue

Le projet est susceptible d'apporter une contribution tangible au développement durable de la région partenaire : il doit démontrer de manière adéquate les bénéfices sociaux, économiques et environnementaux apportés à la communauté dans laquelle il s'insère. L'estimation de ces bénéfices et la description des moyens réservés à l'évaluation sont incluses dans la demande de soutien financier. Le projet doit être en principe réplicable. II fait l'objet d'un rapport final faisant notamment état des enseignements à tirer.

\section{- Cohérence}

Le projet constitue une réponse à une mise en évidence locale des besoins.

- Fonds propres

La contribution du canton de Genève ne peut dépasser $50 \%$ du coût global du projet, le solde est à la charge du porteur de projet (fonds propres, contribution d'autres bailleurs de fonds et/ou de partenaires locaux).

- Viabilité

Le projet doit être viable et viser à une pérennité.

- Impacts attendus

II est attendu des projets soutenus des résultats (sociaux, économiques, environnementaux) identifiables permettant à moyen terme de contribuer à l'autonomie et à l'enrichissement du tissu économique local. 